\title{
Hadron tomography studies by generalized parton distributions and distribution amplitudes
}

\author{
S. Kumano ${ }^{1,2}$ \\ ${ }^{1}$ KEK Theory Center, Institute of Particle and Nuclear Studies, KEK \\ and Department of Particle and Nuclear Physics, Graduate University for Advanced Studies, \\ 1-1, Ooho, Tsukuba, Ibaraki, 305-0801, Japan \\ ${ }^{2}$ J-PARC Branch, KEK Theory Center, Institute of Particle and Nuclear Studies, KEK \\ and Theory Group, Particle and Nuclear Physics Division, J-PARC Center, \\ 203-1, Shirakata, Tokai, Ibaraki, 319-1106, Japan
}

\begin{abstract}
We discuss hadron-tomography studies for the nucleon and exotic hadrons by high-energy hadron reactions. First, the constituent-counting rule is explained for determining internal quark configurations of exotic-hadron candidates by scaling properties of high-energy exclusive cross sections. Next, possibilities are discussed for investigating the generalized parton distributions (GPDs) of the nucleon and exotic hadrons at J-PARC. In particular, we study hadronic $2 \rightarrow 3$ process $p+p \rightarrow N+\pi+B$, exclusive Drell-Yan process, and exotic-hadron GPDs. For determining three-dimensional structure of unstable exotic hadrons, we consider $s-t$ crossed quantities of the GPDs called generalized distribution amplitudes (GDAs), which can be investigated at KEKB. We explain possible studies of the GDAs by two-photon processes.
\end{abstract}

\section{Introduction}

Internal structure of the nucleon has been investigated for a long time by lepton and hadron scattering processes at various energies, and major properties are now understood. However, the origin of the nucleon spin has not been clarified yet although it is one of fundamental physics quantities. For understanding the whole picture of the nucleon spin in terms of quarks and gluons, it is essential to understand partonic orbital-angular-momentum contributions. Therefore, it became necessary to establish three-dimensional (3D) picture of the nucleon, and this field is called hadron tomography. It is now under active investigations through the 3D structure functions of generalized parton distributions (GPDs) and transverse-momentum-dependent parton distributions (TMDs). There are also generalized distribution amplitudes (GDAs) which are $s$ - $t$ crossed quantities to the GPDs.

These 3D structure functions are key quantities in solving the nucleon-spin puzzle. However, it is interesting to use them for exotic-hadron studies because it is not easy to find internal quark-gluon configurations, namely undoubted evidence of exotic nature, solely by low-energy observables such as masses and decay widths. In general, high-energy reactions should be useful for finding internal structure of exotic hadrons by using, for example, fragmentation functions [1], constituent-counting rule $[2,3]$, and the $3 \mathrm{D}$ structure functions [4] because the appropriate degrees of freedom are quark and gluons. 
In this article, possible exotic-hadron studies are proposed by using high-energy reactions, together with the usual nucleon GPDs, at facilities such as J-PARC and KEKB. First, we explain the constituent-counting rule, predicted by perturbative $\mathrm{QCD}$, as a useful guidance to probe the internal quark-gluon configurations of exotic-hadron candidates [2, 3] in Sec. 2. Then, the GPDs for the nucleon and exotic-hadron candidates are discussed in Sec. 3 including possible experimental studies at J-PARC [4-6]. Since there is no stable exotic hadron, the direct GPD studies do not seem to be possible for exotic hadrons, except for transition GPDs such as the ones from the nucleon to an exotic hadron. In this respect, the GDAs could be appropriate 3D structure functions for studying exotic hadrons [4] because an exotic-hadron pair could be produced in the final state even for an unstable hadron. We explain such GDAs in Sec. 4, and it should be a possible project as two-photon processes at KEKB $[4,7]$. We summarize our studies in Sec. 5.

\section{Constituent-counting rule for exotic hadrons}

The constituent-counting rule was predicted in hard exclusive reactions by perturbative QCD. As shown typically in Fig. 1, the two-body hadron reaction $a+b \rightarrow$ $c+d$ with large $p_{T}$ should occur due to hard gluon exchanges between quarks in the hadrons. By considering the hard gluon and quark propagators together with other kinematical factors, we obtain the scaling relation for the cross section as [2]

$$
\frac{d \sigma_{a b \rightarrow c d}}{d t}=\frac{1}{s^{n-2}} f_{a b \rightarrow c d}(t / s),
$$

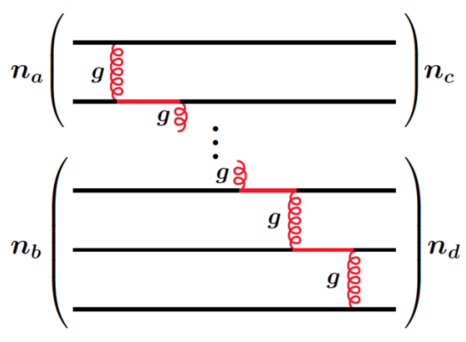

Figure 1. A hard-gluon exchange process [2].

where $s$ and $t$ are Mandelstam variables, $f(t / s)$ is a function depends on the scattering angle, and $n$ is the total number of constituents given by $n=n_{a}+n_{b}+n_{c}+n_{d}$. The high-energy cross section is proportional to $1 / s^{n-2}$ with the number of constituents $(n)$, and it is called the constituent-counting rule. It has been confirmed experimentally by various hard processes in lepton-hadron and hadronhadron exclusive reactions, even for compound systems of hadrons such as the deuteron, ${ }^{3} \mathrm{H}$, and ${ }^{3} \mathrm{He}$. Since it is not easy to judge whether or not discovered hadrons are actually exotic only by low-energy observables, we think that it is appropriate to use high-energy reaction processes to find internal constituents of exotic hadrons. Especially, the appropriate degrees of freedom are quarks and gluons at high energies, so that such approaches could be promising as future exotic-hadron studies.

For example, we investigate an exotic-hadron candidate $\Lambda(1405)$, which is considered to be $\bar{K} N$ molecule or pentaquark state [8], by the constituent counting rule. First, we look at the ground $\Lambda$

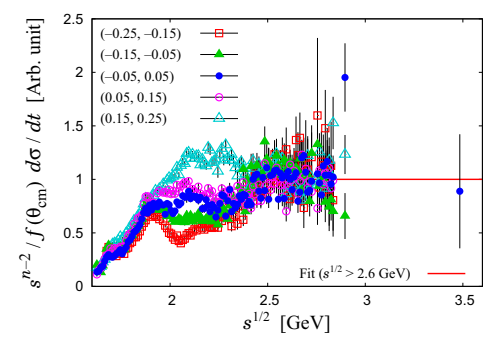

Figure 2. Scaling in $\gamma+p \rightarrow K^{+}+\Lambda[3]$.

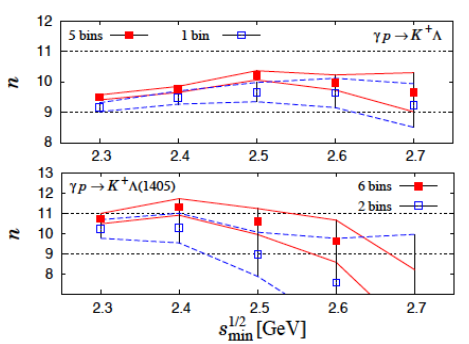

Figure 3. Energy dependence of factor $n[3]$. 
production $\gamma+p \rightarrow K^{+}+\Lambda$, and the cross section scales as shown in Fig. 2. The scaling factor $n$ determined from the data depends the minimum energy $\sqrt{s_{\min }}$ for the scaling region as shown in Fig. 3. The factor $n=9(=1+3+2+3)$ means that the ground $\Lambda$ is consistent with the $q q q$ picture.

There are also data for the $\Lambda(1405)$ production $\gamma+p \rightarrow K^{+}+\Lambda(1405)$. We analyzed its data and obtained the scaling factor $n$ as shown in Fig. 3. The figure implies an interesting tendency that $\Lambda(1405)$ looks like a five-quark state $(\bar{K} N$ molecule or pentaquark state) at relatively-low energies but it becomes three-quark one at high energies. However, the errors of $n$ are too large to draw a solid conclusion at this stage. Future experimental studies including by a $12-\mathrm{GeV}$ JLab experiment are needed.

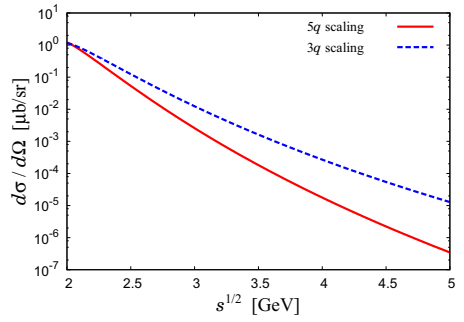

Figure 4. $\pi p \rightarrow K^{0} \Lambda(1405)$ cross sections [2]. Similar studies should be possible by hadron accelerator facilities such as J-PARC. As an example, we estimated the cross sections for $\pi+p \rightarrow K^{0}+\Lambda(1405)$ by taking $n_{\Lambda(1405)}=3$ or 5 . There are large differences between the curves, so that the internal structure of $\Lambda(1405)$ can be investigated through the counting rule at J-PARC.

\section{Generalized parton distributions and J-PARC project}

The GPDs are defined for the nucleon by off-forward nucleon matrix elements of quark (and gluon) bilocal operators as [4-6]

$$
\begin{aligned}
& \left.\int \frac{d y^{-}}{4 \pi} e^{i x P^{+} y^{-}}\left\langle p^{\prime}\left|\bar{q}(-y / 2) \gamma^{+} q(y / 2)\right| p\right\rangle\right|_{y^{+}=\vec{y}_{\perp}=0}=\frac{1}{2 P^{+}} \bar{u}\left(p^{\prime}\right)\left[H^{q}(x, \xi, t) \gamma^{+}+E^{q}(x, \xi, t) \frac{i \sigma^{+\alpha} \Delta_{\alpha}}{2 m_{N}}\right] u(p), \\
& \left.\int \frac{d y^{-}}{4 \pi} e^{i x P^{+} y^{-}}\left\langle p^{\prime}\left|\bar{q}(-y / 2) \gamma^{+} \gamma_{5} q(y / 2)\right| p\right\rangle\right|_{y^{+}=\vec{y}_{\perp}=0}=\frac{1}{2 P^{+}} \bar{u}\left(p^{\prime}\right)\left[\tilde{H}^{q}(x, \xi, t) \gamma^{+} \gamma_{5}+\tilde{E}^{q}(x, \xi, t) \frac{\gamma_{5} \Delta^{+}}{2 m_{N}}\right] u(p),
\end{aligned}
$$

where $\sigma^{\alpha \beta}$ is $\sigma^{\alpha \beta}=(i / 2)\left[\gamma^{\alpha}, \gamma^{\beta}\right]$, gauge-link operators for the gauge invariance are not explicitly written, $H^{q}(x, \xi, t)$ and $E^{q}(x, \xi, t)$ are the unpolarized quark GPDs, and $\tilde{H}^{q}(x, \xi, t)$ and $\tilde{E}^{q}(x, \xi, t)$ are the polarized ones. By the momentum assignments in Fig. 5, the variables $x$ and $\xi$ are defined by

$$
\begin{aligned}
& x=\frac{((k-\Delta / 2)+(k+\Delta / 2))^{+}}{\left(p+p^{\prime}\right)^{+}}=\frac{k^{+}}{P^{+}}, \\
& \xi=\frac{\left(p-p^{\prime}\right)^{+}}{\left(p+p^{\prime}\right)^{+}}=\frac{-\Delta^{+}}{2 P^{+}}=\frac{((k-\Delta / 2)-(k+\Delta / 2))^{+}}{\left(p+p^{\prime}\right)^{+}},
\end{aligned}
$$

so that $x$ and $2 \xi$ are the light-cone momentum fractions of the average momentum and momentum transfer for the relevant quarks to the average momentum of the parent nucleon. The

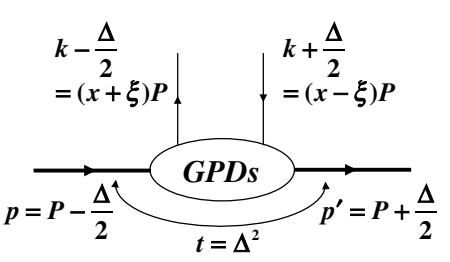

Figure 5. Kinematics of GPDs [6]. range of $x$ is from -1 to 1 , whereas $\xi$ is from 0 to 1 . The GPDs have necessary information to find 3D structure of the nucleon, and they are key quantities in the studies of hadron tomography. There are three important features in the GPDs. (I) In the forward limit ( $\Delta \rightarrow 0, \xi \rightarrow 0$ and $t \rightarrow 0)$, they become unpolarized and longitudinally-polarized parton distribution functions (PDFs) for the nucleon: $H^{q}(x, 0,0)=q(x)$ and $\tilde{H}^{q}(x, 0,0)=\Delta q(x)$. (II) The first moments of the unpolarized GPDs are Dirac and Pauli form factors of the nucleon, and those of the polarized GPDs are axial and pseudoscalar form factors:

$$
\int_{-1}^{1} d x H^{q}(x, \xi, t)=F_{1}^{q}(t), \int_{-1}^{1} d x E^{q}(x, \xi, t)=F_{2}^{q}(t), \int_{-1}^{1} d x \tilde{H}^{q}(x, \xi, t)=g_{A}^{q}(t), \int_{-1}^{1} d x \tilde{E}^{q}(x, \xi, t)=g_{P}^{q}(t) .
$$


(III) The second moment of a GPD combination becomes the quark total angular momentum: $J^{q}=$ $\int_{-1}^{1} d x x\left[H^{q}(x, \xi, t=0)+E^{q}(x, \xi, t=0)\right] / 2$, which makes it possible to find the the quark orbitalangular-momentum contribution to the nucleon spin by $J^{q}=\Delta q / 2+L^{q}$. We now explain how the GPDs can be investigated at hadron facilities such as J-PARC in addition to the usual deeply virtual Compton scattering (DVCS) in the following subsections.

\subsection{Generalized parton distributions in $p+p \rightarrow N+\pi+B$}

The GPDs have been experimentally investigated mainly in the DVCS. However, it is possible to study them in hadron reactions. Hadron-reaction rates are generally larger than the electromagnetic Compton scattering, so that extended kinematical regions could be probed. Furthermore, it is a unique opportunity that the interesting Efremov-Radyushkin-Brodsky-Lepage (ERBL) region can be studied in the reaction $N+N \rightarrow N+\pi+B$ of Fig. 6 [5]. There are three kinematical regions in the GPDs as shown

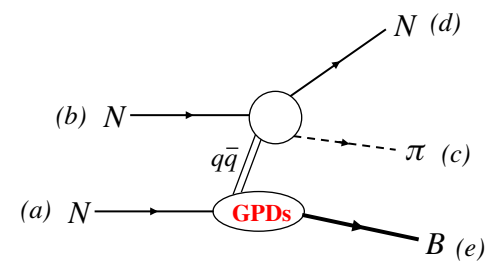

Figure 6. $N N \rightarrow N \pi B$ process for GPDs. in Fig. 7. The regions $(a)$ and $(c)$ are the DGLAP (Dokshitzer-Gribov-Lipatov-Altarelli-Parisi) regions, and their GPDs corresponds to the antiquark and quark distributions, respectively. The $(b)$ is the ERBL region, and the GPDs of this region indicate quark-antiquark distributions.

We define the Mandelstam variables as $s^{\prime}=\left(p_{c}+p_{d}\right)^{2}, t^{\prime}=\left(p_{b}-p_{d}\right)^{2}$, and $u^{\prime}=\left(p_{b}-p_{c}\right)^{2}$. For investigating the GPDs, we consider the hard region $s^{\prime},\left|t^{\prime}\right|,\left|u^{\prime}\right| \gg m_{N}^{2}$. In the reaction $N+N \rightarrow$ $N+\pi+B$ of Fig. 6, it is possible to probe the ERBL region. We estimated its cross sections for J-PARC kinematics of $30 \mathrm{GeV}$ and $50 \mathrm{GeV}$ proton-beam energies in Fig. 8 by taking $t=\left(p_{a}-p_{e}\right)^{2}=-0.3$ $\mathrm{GeV}^{2}$ and $t^{\prime}=-5.0 \mathrm{GeV}^{2}$ as an example [5]. Here, the neutron or $\Delta^{0}$ are assumed for the final-state baryon $B$, and the pion- and $\rho$-pole terms are used for the ERBL GPDs. These cross-section estimates are intended for proposing a future J-PARC experiment. The GPDs, $H, E, \tilde{H}$, and $\tilde{E}$, for the nucleon and the $N \rightarrow \Delta$ transition are involved in the cross section, and they are theoretically estimated by the meson-pole contributions. The interactions for $\pi N \rightarrow \pi N$ and $\rho N \rightarrow \pi N$, which correspond to the upper part of Fig. 6, are determined from the measurements of the BNL-E838 experiment [5]. The hadron facility measurements at J-PARC and possibly at GSI-FAIR should provide new information on the GPDs in the unique ERBL region. In order to determine the GPDs for the studies of the nucleon spin and 3D structure, all the kinematical regions should be investigated, so that the J-PARC measurement is valuable and complementary to the usual DVCS experiments.

(a)

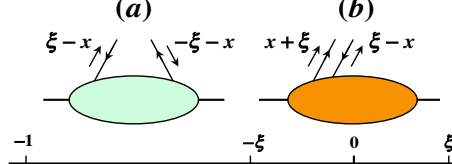

$-1<x<\xi(x+\xi<0, x-\xi<0) \quad \xi<x<1(x+\xi>0, x-\xi>0)$

$-\xi<x<\xi(x+\xi>0, x-\xi<0)$

Figure 7. Three kinematical regions of GPDs: $(a)$ emission and reabsorption of an antiquark, $(b)$ emission and a quark and an antiquark, $(c)$ emission and reabsorption of a quark. (c)

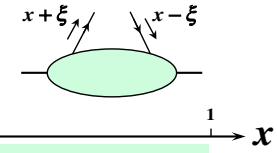




\subsection{Generalized parton distributions in exclusive Drell-Yan}

There is another possibility to investigate the GPDs at JPARC by using an exclusive Drell-Yan process $\pi^{-}+p \rightarrow$ $\mu^{+} \mu^{-}+n[6]$ because high-momentum unseparated-hadron (essentially pion) beamline will become available soon. A typical GPD process for the exclusive dimuon production is shown in Fig. 9, where the process is described by the GPDs combined with the pion distribution amplitude (DA). However, the distribution amplitude should be constrained by other measurements such as on the $\gamma \rightarrow \pi$ transition form factor of the Belle and BaBar experiments, although

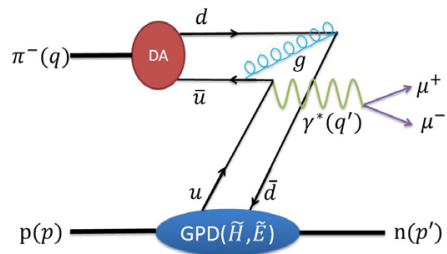

Figure 9. GPD contribution to exclusive Drell-Yan [6]. there are still some ambiguities whether it is the asymptotic form, the Chernyak-Zhitnitsky one, or something in between.

The GPDs $\tilde{H}$ and $\tilde{E}$ contribute to the cross section, and a typical GPD parametrization GK2013 is used for showing numerical results in Fig. 10, where three possible pion momenta, $p_{\pi}=10,15$, and $20 \mathrm{GeV}$, are taken and $t_{0}$ is the limiting value of the Mandelstam variable $t$ at the zero scattering angle. This estimate is intended for a J-PARC proposal, and actual GPDs should be determined from future measurements at J-PARC together with other data including DVCS ones. Next, Monte Carlo simulations of missing-mass $\left(M_{X}\right)$ spectra of $\mu^{+} \mu^{-}$events are shown in Fig. 11 . There are contributions from various sources: exclusive Drell-Yan (red), inclusive Drell-Yan (blue), $\mathrm{J} / \psi$ (green), and random background (purple). It is obvious that the exclusive Drell-Yan dominates the events at $M_{X} \simeq 1 \mathrm{GeV}$, so that it is a feasible experiment at J-PARC. At JLab, there is a proposed experiment to measure the opposite process $\gamma^{*} N \rightarrow \pi N$; however, it is a timelike photon process at J-PARC and the probed $x$ region is smaller than the JLab case [6], so that the J-PARC experiment is a valuable complementary contribution to the GPD studies.

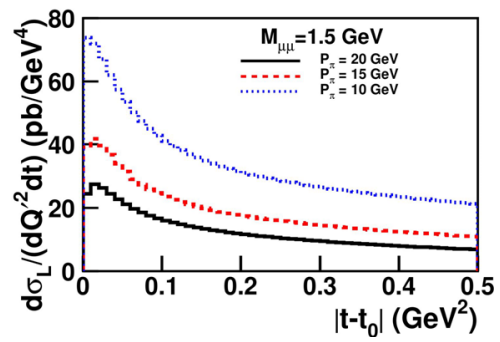

Figure 10. $t$ dependence of $\pi^{-}+p \rightarrow \mu^{+} \mu^{-}+n$ cross sections for J-PARC kinematics [6].

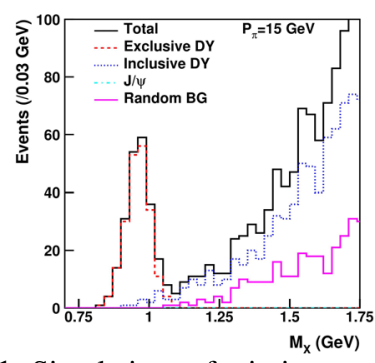

Figure 11. Simulations of missing-mass $\left(M_{X}\right)$ spectra of $\mu^{+} \mu^{-}$events [6].

\subsection{Generalized parton distributions for exotic hadrons}

In addition to the constituent counting rule in Sec. 2, the GPDs, in general the 3D structure functions, can be used for exotic-hadron studies [4] because the relevant degrees of freedom are quarks and gluons at high energies and because exotic signatures should appear in the 3D structure. The GPDs have two types of information on internal structure of a hadron: (1) longitudinal-momentum distributions, namely PDFs $\left(f_{n}(x)\right)$, (2) transverse form factors $\left(F_{n}^{h}(t, x)\right)$ which also depend on the longitudinalmomentum fraction $x$. Therefore, the GPDs could be expressed by their multiplication as a simple form:

$$
H_{q}^{h}(x, \xi=0, t)=f_{n}(x) F_{n}^{h}(t, x)
$$


where $n$ indicates the number of valence quarks.

A simple $x$-dependent form of the longitudinal PDFs is $f_{n}(x)=C_{n} x^{\alpha_{n}}(1-x)^{\beta_{n}}$. We consider valence-quark distributions in this form. Then, if $\beta_{n}$ is known, the parameters $C_{n}$ and $\alpha_{n}$ are determined by the valence-quark number $\int_{0}^{1} d x f_{n}(x)=n$, and the quark momentum $\int_{0}^{1} d x x f_{n}(x)=\langle x\rangle_{q}$. The parameter $\beta_{n}$ is associated with the functional behavior in the elastic limit $x \rightarrow 1$, and it is determined by the constituent counting rule as $\beta_{n}=2 n-3+2 \Delta S_{z}$ with the spin factor $\Delta S_{z}=\left|S_{z}^{q}-S_{z}^{h}\right|$. From these three conditions, we theoretically obtain the PDFs not only for the ordinary hadrons, such as pion and nucleon, but also for exotic tetraquark and pentaquark hadrons, and the results are shown by the solid curves in Fig. 12. In comparison, typical PDFs of the pion and the proton extracted from experimental measurements are shown by the dotted curves at $Q^{2}=2 \mathrm{GeV}^{2}$. Our theoretical curves and the PDF parametrizations agree with each other. Therefore, the predications for the new PDFs of the tetraquark and pentaquark hadrons should be interesting if they are measured by experiments.

Next, we explain that the transverse form factor $F_{n}^{h}(t, x)$ also has information on exotic nature [4]. A simple form of this function is an exponential form $F_{n}^{h}(t, x)=e^{(1-x) t /\left(x \Lambda^{2}\right)}$, where $\Lambda$ is cutoff parameter for the transverse momentum and it is related to the the root-mean-square radius by $\left\langle r_{\perp}^{2}\right\rangle=$ $4(1-x) /\left(x \Lambda^{2}\right)$. Since the pion and nucleon form factors are roughly given by the monopole and dipole forms $\left(1 /\left(|t|+\Lambda^{2}\right), 1 /\left(|t|+\Lambda^{2}\right)^{2}\right)$, the Gaussian form could be too steep as a function of $q_{\perp}^{2}$ and it is realized only in light nuclei. We do not step into such details in this article. The results are shown in Fig. 13 for $x=0.2$ and 0.4. Depending on compact $q \bar{q} / q q q$ hadrons or diffuse molecular exotic hadrons, the transverse form factors are different, so it should be an appropriate quantity to find the internal structure of exotic-hadron candidates.

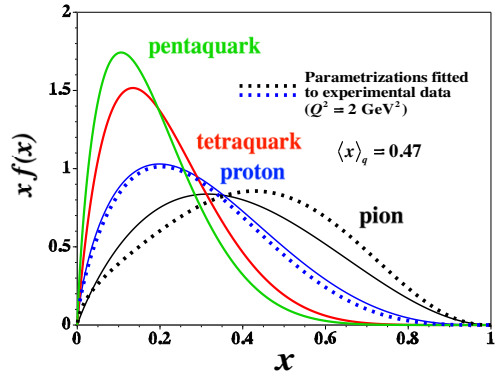

Figure 12. Valence-quark distributions for pion, proton, tetraquark, and pentaquark [4].

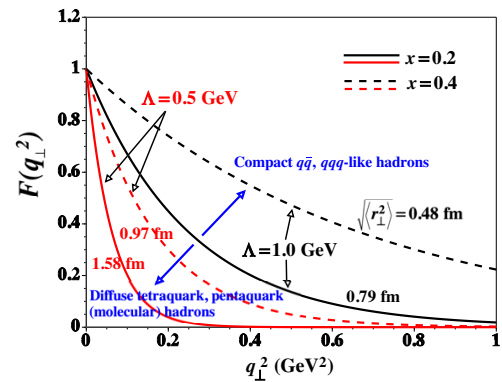

Figure 13. Transverse form factors at $x=0.2,0.4$ [4].
The GPDs of the exotic hadrons could not be observed directly at least at this stage because the exotic hadrons cannot exist as stable fixed targets. Of course, transition GPDs such as for proton $\rightarrow$ an exotic hadron can be investigated in future. For example, by using high-momentum kaon beam which will become available at J-PARC, it is possible to investigate the transition GPDs of $p \rightarrow \Lambda$ (1405) as shown in Fig. 14, and they should reflect the exotic nature of $\Lambda(1405)$. Since the transition GPDs, especially on exotic hadrons, are not well studied, theoretical and experimental efforts are needed to push this kind of experimental projects in future.

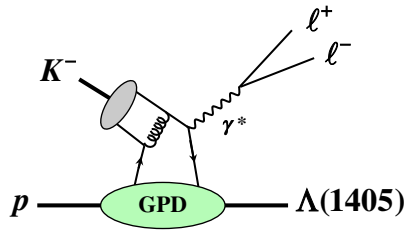

Figure 14. Transition GPDs at J-PARC. 


\section{Generalized distribution amplitudes and KEKB project}

It is an interesting idea to use the transition GPDs for exotic-hadron studies; however, another promising way of studying 3D structure functions for exotic hadrons is to use general distribution amplitudes (GDAs) because exotic hadrons can be produced in s-channel processes [4]. A two-photon process to produce an exotic-hadron pair $\gamma \gamma^{*} \rightarrow h \bar{h}$ is shown in Fig. 15. If the kinematical condition, $Q^{2}\left(=-q^{2}\right) \gg W^{2}\left(=\left(p+p^{\prime}\right)^{2}\right), \Lambda^{2}$, is satisfied, we have the factorization of the process in terms of the hard photon interaction and the GDAs. This two photon process is the $s$ - $t$ crossed one to the DVCS $\left(\gamma^{*} h \rightarrow \gamma h\right)$ where the GPDs are investigated, so that the $s-t$ crossed

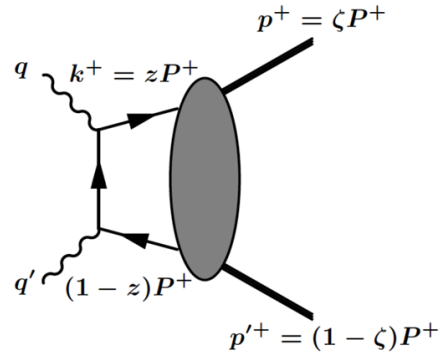

Figure 15. $\gamma \gamma \rightarrow h \bar{h}$ and GDAs [4]. 3D structure functions of the GPDs are the GDAs.

The quark GDAs are defined by the same lightcone operator to the GPDs between the vacuum and the hadron pair $h \bar{h}$ :

$$
\Phi_{q}^{h \bar{h}}(z, \zeta, s)=\left.\int \frac{d y^{-}}{2 \pi} e^{i(2 z-1) P^{+} y^{-}}\left\langle h(p) \bar{h}\left(p^{\prime}\right)\left|\bar{\psi}(-y / 2) \gamma^{+} \psi(y / 2)\right| 0\right\rangle\right|_{y^{+}=\vec{y}_{\perp}=0},
$$

and the gluon GDA is defined in the similar way. The $s$ - $t$ crossing relates the GDAs to GPDs, and the relation is given by

$$
\Phi_{q}^{h \bar{h}}\left(z, \zeta, W^{2}\right) \longleftrightarrow H_{q}^{h}\left(x=\frac{1-2 z}{1-2 \zeta}, \xi=\frac{1}{1-2 \zeta}, t=W^{2}\right)
$$

It means that the GDAs contain the information of GPDs not only in the usual physical region but also in the unphysical regions: $0 \leq|x|<\infty, 0 \leq|\xi|<\infty,|x| \leq|\xi|$, and $t \geq 0$. A simple functional form is given by

$$
\Phi_{q}^{h \bar{h}(I=0)}\left(z, \zeta, W^{2}\right)=N_{h(q)} z^{\alpha}(1-z)^{\beta}(2 z-1) \zeta(1-\zeta) F_{h(q)}\left(W^{2}\right),
$$

where $F_{h(q)}\left(W^{2}\right)$ is a form factor of the quark part of the energy-momentum tensor. According to the constituent counting rule, it is expressed as $F_{h(q)}\left(W^{2}\right)=1 /\left[1+\left(W^{2}-4 m_{h}^{2}\right) / \Lambda^{2}\right]^{n-1}$, where the factor $n$ is $n=2$ for ordinary $q \bar{q}$ mesons and $n=4$ for tetraquark hadrons.

The $f_{0}(980)$ and $a_{0}(980)$ mesons have been controversial hadrons which seem to be tetraquark states or $K \bar{K}$ molecules [9]. Here, we show $W^{2}$ dependence of the cross section $e \gamma \rightarrow e^{\prime} h \bar{h}$ in Fig. 16 for $h=f_{0}(980)$ or $a_{0}(980)$ by using the simple GDAs in Eq. (8) with the form factor suggested by the constituent counting rule. There are large differences in the cross sections depending on the constituent number $n, n=2$ (ordinary meson, $q \bar{q}$ ) or $n=4$ (tetraquark type, $q q \bar{q} \bar{q})$. Even for the $n=4$ hadron, it is possible distinguish a compact $q q \bar{q} \bar{q}$ state from a diffuse molecular state

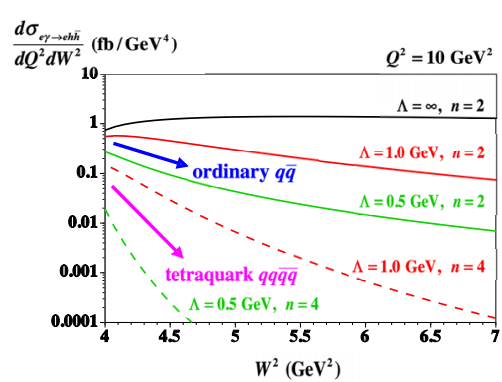

Figure 16. Cross section for $h \bar{h}$-pair production [4]. from the measurement. Therefore, the GDAs should provide valuable information on internal structure of exotic hadrons, and such two-photon physics is certainly a possible project at KEKB [7]. 


\section{Summary}

It became crucial to investigate the 3D structure functions for finding the original of nucleon spin including partonic orbital-angular-momentum contributions. One of such 3D structure functions is the GPDs which have been investigated by the DVCS so far. However, it is also possible to investigate them at hadron facilities such as J-PARC, for example, by using the $2 \rightarrow 3$ process $p+p \rightarrow N+\pi+B$ and the exclusive Drell-Yan process $\pi^{-}+p \rightarrow \mu^{+} \mu^{-}+n$. In addition, the 3D structure functions, the GPDs and GDAs, can be used for finding internal structure of exotic hadrons. In particular, the GDAs should be appropriate 3D quantities for the exotic-hadron studies because the exotic hadrons can be created in the final state, whereas it is difficult to measure the exotic-hadron GPDs directly because unstable exotic hadrons cannot be used as stable fixed targets. The GDAs can be investigated by the two-photon processes, for example, at KEKB and it is a new promising direction of exotic-hadron studies.

\section{Acknowledgements}

This work was supported by Japan Society for the Promotion of Science (JSPS) Grants-in-Aid for Scientific Research (KAKENHI) Grant No. JP25105010.

\section{References}

[1] M. Hirai, S. Kumano, M. Oka, and K. Sudoh: Phys. Rev. D 77, 017504 (2008).

[2] H. Kawamura, S. Kumano, and T. Sekihara, Phys. Rev. D 88, 034010 (2013).

[3] W.-C. Chang, S. Kumano, and T. Sekihara, Phys. Rev. D 93, 034006 (2016).

[4] H. Kawamura and S. Kumano, Phys. Rev. D 89, 054007 (2014).

[5] S. Kumano, M. Strikman, and K. Sudoh, Phys. Rev. D 80, 074003 (2009).

[6] T. Sawada, W.-C. Chang, S. Kumano, J.-C. Peng, S. Sawada, and K. Tanaka, Phys. Rev. D 93, 114034 (2016).

[7] S. Kumano and Q.-T. Song, research in progress.

[8] T. Sekihara and S. Kumano, Phys. Rev. C 89, 025202 (2014).

[9] S. Kumano and V. R. Pandharipande, Phys. Rev. D 38, 146 (1988); F. E. Close, N. Isgur, and S. Kumano, Nucl. Phys. B 389, 513 (1993); T. Sekihara and S. Kumano, Phys. Rev. D 92, 034010 (2015). 